\title{
Article
}

\section{The Development of an Online Standalone Cognitive Hazard Training for Laparoscopic Cholecystectomy: A Feasibility Study}

Isreb, Siddek, Attwood, Stephen, Hesselgreaves, Hannah, Mclachlan, John Charles and Illing, Jan

Available at http://clok.uclan.ac.uk/29928/

Isreb, Siddek, Attwood, Stephen, Hesselgreaves, Hannah, Mclachlan, John Charles ORCID: 0000-0001-5493-2645 and Illing, Jan (2020) The Development of an Online Standalone Cognitive Hazard Training for Laparoscopic

Cholecystectomy: A Feasibility Study. Journal of Surgical Education, 77 (1). pp. 1-8. ISSN 1931-7204

It is advisable to refer to the publisher's version if you intend to cite from the work. http://dx.doi.org/10.1016/j.jsurg.2019.09.002

For more information about UCLan's research in this area go to http://www.uclan.ac.uk/researchgroups/ and search for < name of research Group>.

For information about Research generally at UCLan please go to http://www.uclan.ac.uk/research/

All outputs in CLoK are protected by Intellectual Property Rights law, including Copyright law. Copyright, IPR and Moral Rights for the works on this site are retained by the individual authors and/or other copyright owners. Terms and conditions for use of this material are defined in the policies page. 
The development of an online standalone cognitive hazard training for laparoscopic cholecystectomy: a feasibility study

Siddek Isreb a, Stephen Attwood ${ }^{\text {b }}$, Hannah Hesselgreaves ${ }^{\text {c }}$, Professor John McLachlan ${ }^{\text {d, Professor }}$ Jan Illing e

a) Corresponding author, $\mathrm{PhD}$ in medical education from Durham University and member of the Faculty of surgical Trainers at the Royal College of Surgeon of Edinburgh, Postal address C/O Professor Jan Illing office Newcastle University, Framlington Place, Newcastle upon Tyne, NE2 4HH, UK, Siddek.Isreb@nhs.net

b) Honorary Professor at Durham University, Department of Health Services Research, Queen's Campus, Stockton on Tees, Durham, UK seaattwood@gmail.com

c) Associate Professor at Northumbria University, Newcastle Business School, Newcastle upon Tyne, NE1 8ST, UK hannah.hesselgreaves@northumbria.ac.uk

d) Professor at University of Central Lancashire, Preston, PR1 2HE, UK JCMcLachlan1@uclan.ac.uk

e) Professor of medical education, Newcastle University, Framlington Place, Newcastle upon Tyne, NE2 4HH, UK Jan.Illing@newcastle.ac.uk

Key words: Non-Technical Skills for Surgeons, Surgery Simulation, Surgical Competency, Patient Safety, e-Learning Resource, Student Cognition

\section{Summary declaration of interest:}

1. Northumbria Healthcare NHS foundation trust: Grant used to buy the laptop and the software used to process the audio-visual files.

2. Durham University: this project was part of a $\mathrm{PhD}$ research and the university hosted the online module on their website as part of the university $\mathrm{PhD}$ support services. 


\begin{abstract}
Introduction

In the UK, surgical training is under pressure due to reductions in training time and training opportunities, which pose patient safety risks. Cognitive, non-technical, training has been suggested as a possible solution inspired by the identified benefits in aviation industry. A recent review article highlighted the need for such training despite its high cost and the need for expert trainers.
\end{abstract}

\title{
Aim
}

This study aimed to design and test the feasibility of an online standalone module to address the current gap in cognitive surgical training.

\section{Method}

An online standalone, Cognitive Hazard Training module for laparoscopic cholecystectomy was created. It combined multiple choice questions, extended matching items, and single-line free text questions. It contained relevant sketch images and real life hazards video clips, highlighting potential mistakes to enhance: safety knowledge, reduce bias, and improve self-limitation awareness.

Two experts were invited to validate the prototype before testing its feasibility in one English Deanery training environment.

\section{Results}

In total 93 candidates signed up to review the training. However only 47 (50\%) later participated and 33 completed the Module. Those included 3 juniors, 20 higher trainees and 10 consultants. Candidates' answers were quantitatively analysed. Qualitative feedback was also collected from 27 candidates, via semistructured interviews.

The overall feedback from the feasibility study was positive. Results supported this online resource value in enhancing knowledge and awareness. Interview data also suggested the module's potential to change trainees' practice by being more cautious and adhering to the safety steps of dissection.

\section{Discussion}

This new training module overcomes some of the previously reported problems in surgical cognitive training. It is a stand-alone online resource with low running cost and does not require expert trainers. The feasibility study supported the aim to enhance hazard awareness and create an attitude shift towards adherence to safety steps during the procedure.

\section{ACGME competency: Practice-Based Learning and Improvement}




\section{Introduction:}

Surgical training is under great pressure to maintain standards while reducing training times especially after the implementation of the European Working Time Directive (EWTD) (1,2). The impact of this means that trainees will have to reach competency within a fifth of the previously recommended training time (1) and with only two-thirds of the previously recommended number of training operations (3). To add to this pressure, surgical practice is expanding. This is a result of new technologies and surgical procedures such as laparoscopic surgery described as the biggest unaudited expansion in surgical practice history (4).

The challenge of increasing surgical skills, while reducing training time is clear. Two-thirds of consultant surgeons surveyed expressed deep concern over the future standard of consultant surgeons following such a shortened training programme (5). This concern directed interest at a comparison between surgery and aviation, two hazardous industries, and simulation training has been considered as a possible solution to replace the reduced training time (6).

However, surgical practice is a combination of operative competency and cognitive (non-technical) skills such as decision making and communication. In fact, cognitive skills are the main component in surgical safety. This has been clearly illustrated when analysing the surgical errors of fully qualified and technically competent surgeons (7). Evidence has linked avoidable deaths to surgeons' false perceptions of their own ability, which was clearly emphasised following the Bristol Royal Infirmary case (8). The same effects were also seen in aviation safety. Cognitive factors resulting from pilots' incorrect assessment of risk are the driving force behind the majority of fatal accidents (9). In other words, safety cannot be reached by the mere focus on motor surgical skills training. Cognitive training plays an important role in enhancing surgical safety and reducing patient harm.

\section{$\underline{\text { Cognitive training principles }}$}

Kahneman (10) described a putative two system model operating constantly within our heads. System One provides the quick thinking, easy judgement and superficial and sometimes even bias information analysis, while System Two deals with the deep thinking and reasoning. Unlike System One, System Two consumes a lot of energy and a major share of the limited brain resources. Aware of such limited resources, System Two prioritises the use of those resources and engages only in the case of high demand such as important decisions and deep thinking activity. As a result, System One is the default system in use. It operates automatically and constantly under the lax supervision of System Two.

System Two can programme System One to perform skilled actions and judgements after adequate training. Such training moves the demand from System Two to System One and facilitates the transition from difficult, tunnel-vision novice in-training to easy automatic expert practice. System Two can also programme System One to look for certain patterns and raise attention to their presence and importance. An example of such training is present in rapid hazard recognition while driving. Such training reduces errors 
and enhances skill acquisition. This principle is the base for cognitive hazard training implemented in military pilot training (11).

However, due to self-motivation bias humans view others' mistakes in an objective way, but struggle to realise their own errors (12). As a result Dror (13) suggested an intermediate phase of error recognition in others using interactive video-clips to provide informative feedback in a similar manner to the hazard perception clips used in the UK driving test. The latter is part of the UK theory computer-based driving test. It includes fourteen video-clips of everyday road scenes with developing hazards. Those are situations where drivers are forced to take an action to avoid potential hazards such as slowing down on spotting a flashing indicator of a parked car starting to move away. Driving test candidates are asked to click the mouse when they spot such situations.

Dror's suggested training clips progress from simple exaggerated mistakes to more subtle errors. Trainees have to identify the possible recovery plans at the end of the process, after being offered such plans earlier in the training. This would eventually help trainees to recognise their own mistakes and reduce them (13).

\section{Cognitive surgical training}

Applying this theory in surgical training provides valuable insight. Training requires a standardised or semistandardised environment with immediate corrective feedback and adequate practice opportunities. It also requires mental programming to identify hazard patterns and initiate mental warnings. Such training explains the importance of hazard perception videos in the UK driving test and its applicability in surgical skills training. As training progresses, System One takes over and performs the skilled tasks, alerting System Two to kick-in, only if pre-trained hazard patterns are identified. Cognitive training to identify possible hazards, would fast track surgical training by providing this important training outside theatre. Cognitive hazard training would also enhance patient safety and focus theatre training on gaining practice. Trainees in theatre will concentrate on sharpening their pre-acquired cognitive skills and practise the remaining cognitive and technical skills to perfection.

Wallace et al (14) conducted a literature review of cognitive training and its adaptation to surgical education. The review article established the value of cognitive training and recommended implementing such training in the surgical curriculum. However, they identified multiple gaps in the available literature with two major limitations. Firstly, the majority of studies were conducted using simulation training rather than being in real life. This made it challenging for the authors of the review article to assess the feasibility of delivering cognitive training as a formal training curriculum component. Secondly, most studies used trained instructors to deliver the cognitive training, rendering such training costly to run and difficult to standardise due to normal style and delivery variation between facilitators.

\section{Aims}


The aim of this study was to develop a cognitive training module that would overcome the limitations of high running cost and variable training delivery. The study also aimed to test the feasibility of cognitive training in the UK surgical training environment.

\section{Methods}

This study was conducted as part of a design-based study in medical education (15). The first step was to create the new cognitive hazard training module to overcome the gaps in cognitive surgical training. The module was planned as a dedicated stand-along online resource to deliver cognitive training without the need for instructors. Such a design would reduce running cost and standardise training delivery. The module should also be procedure specific as the majority of hazards and errors are unique to each surgical procedure.

The design was developed for laparoscopic cholecystectomy, as it is a common general surgical procedure performed by a variety of surgeons. As this procedure is carried out laparoscopically, surgeons rely on the laparoscopic camera to capture the intra-abdominal view and present the video on the laparoscopic stack monitor. This video output on the monitor has a two-dimensional presentation of the intra-abdominal threedimensional environment. Surgeons have to interpret this two-dimensional view and manoeuvre the instruments in the three-dimensional real environment inside the patient's abdomen. In this setting, surgeons cannot have real views as would be the case in open surgery, and laparoscopic views become the only reality to assess the situation, detect the hazards and deal with those hazards throughout the procedure. Missing hazard clues might result in clipping or cutting the wrong structure with resulting complications. In this sense, presenting videos and laparoscopic images over a computer screen would represent a real experience similar to the views seen during the operation. This enables cognitive online hazard training to reach high levels of validity by mimicking real life training.

\section{$\underline{\text { Material and design }}$}

The module contained a combination of multiple choice questions (MCQs), extended matching items (EMIs), and single-line free text questions. It had anatomical and laparoscopic drawings as well as realoperation videos and images. Question topics were selected after multiple consultations rounds with the upper gastroenterology surgeons at Northumbria Health care foundation NHS Trust. This process was supervised by the surgical professor in the team. Module contents were selected to maximise the educational outcome of the cognitive training module and a thorough internet search provided enough materials to cover all agreed topics. Those included laparoscopic visualization of common anatomical variations (16), as well as common risks and dangerous mistakes made during laparoscopic cholecystectomy. Videos were selected after a thorough review of publicly available laparoscopic cholecystectomy operation videos on You Tube. Permissions were obtained from the copyright holder and YouTube to download and process the material under the supervision of Durham University legal department. NHS ethical approval was also obtained 
accordingly. Short hazard clips to represent the imminent dangers were extracted from the full operation videos using Windows Movie Maker 2012.

To facilitate cognitive training, the hazard training module progressed from simple questions to complete case management scenarios. It was also divided into four main sections to signpost trainees and reduce the burden of shifting candidates' attention between various topics. Such mental shifting could lead to tiredness and reduce information retention as a result of the mental overload (10). Such mental overload would counteract the intended cognitive training benefits. Each main section is divided into multiple sub-sections over several screens to provide concentrated repeated training, with immediate feedback after each screen, to facilitate System One training as was recommended by Kahneman (10).

Multiple checks were carried out by supervisors and colleagues to check the content, spell and sense check the questions and ensure the shortened clips' clarity, prior to the final draft. This draft, along with the processed images and videos, was then uploaded onto the Durham University website. The module was presented to the candidates as an assessment to attract their attention, increase engagement and maximise cognitive training. It was accessed by invitation via candidate specific username and password.

The module was piloted using two consultant surgeons, outside the intended feasibility testing area of the Northern Deanery, and fine-tuned accordingly. Full details of the module design process and screenshots of all the module contents are available in the $\mathrm{PhD}$ thesis at Durham university website (15).

\section{$\underline{\text { Recruitment }}$}

The Online Hazard Training Module was presented to all candidates in the surgical department with no exclusions, given that they were involved in Laparoscopic cholecystectomy operations. The researcher presented the design at the general surgical department meetings in the recruitment sites. An invitation email with a username and password, was sent to all candidates who had shown an interest by providing their email addresses after the presentation. Invitations were emailed to 93 candidates. This included 13 foundation doctors (F1 and F2), 37 consultants and 43 specialty registrar (SPR) level doctors (Table 1). Those represent the levels in post graduate training in the UK with doctors progressing after graduation to do two years of foundation training with a combination of medical and surgical placement followed by two years of core surgical training and then six years of general surgical speciality training (SPR) before becoming a consultant general surgeon.

The 43 SPR were divided into three categories depending on their training status and training level. SPRs with a national training number were divided into SPR1 (first three years) and SPR 2 (last 3 years) to represent their level at the national training programme. Doctors without a national training number were grouped as staff-grades for analysis purposes as this group may have a variety of surgical experience. Dissemination was achieved from Durham University, via Blackboard. The Blackboard is a University website which facilitates interaction anonymously but can group the results into the predefined groups to facilitate analysis. Results were downloaded from Blackboard on an excel sheet and manually marked and 
analysed in line with the research aim. This online module was not designed as a real assessment or test hence a detailed individual question validation and analysis, for the online module, is beyond the scope of this research. It aimed at training candidates to pick up the hazard clues and generate hazard avoidance or a mitigation plans. It was presented as an assessment to enhance concentration and engagement.

To support feedback on the model, semi-structure interviews were conducted following completion of the model. In total 27 interviews were conducted to reach data saturation point (17). Effort was made to target representation from all groups and subgroups (Table 1) and ensure feedback was achieved from the full range of surgeons who would be involved in this procedure, such as the educationally active members of the training committee. Laparoscopic cholecystectomy procedures are usually carried out or supervised by general surgeons with an Upper Gastroenterology surgical interest. Occasionally this procedure is carried out by vascular or colorectal consultants, hence these surgeons were also targeted to widen participation.

Semi-structured interviews were conducted face to face, to facilitate greater insight into candidates' experience with the module. Interviews were audio-recorded, professionally transcribed and thematically analysed following the six phases described by Braun and Clark (17). Content thematic analysis was carried out by the $\mathrm{PhD}$ researcher and checked by the supervisory group. This analytical approach produced themes which reflected the evaluative aims of the research (theory-driven); as well as themes that emerged from the data (data-driven). Module answer analysis and quantitative interview analysis results were triangulated to gain further insight into the cognitive hazard training module's feasibility.

\begin{tabular}{|l|c|c|c|c|c|c|}
\hline & Junior & SPR1 & SPR2 & SG & Consultants & Total \\
\hline Invited & 13 & 15 & 21 & 7 & 37 & 93 \\
\hline $\begin{array}{l}\text { Link never } \\
\text { opened }\end{array}$ & 7 & 6 & 7 & 1 & 20 & 41 \\
\hline Introductory & 0 & 0 & 2 & 1 & 2 & 5 \\
\hline page only & 6 & 9 & 12 & 5 & 15 & 47 \\
\hline Started & 3 & 7 & 10 & 3 & 10 & 33 \\
\hline Finished & 2 & 6 & 8 & 2 & 9 & 27 \\
\hline Interviewed & & & & & & \\
\hline
\end{tabular}

Table 1: Cognitive hazard training online module candidates' dissemination, progress and interview distribution.

\section{Results}


Out of the invited 93 candidates who expressed interest in using the module, 41 did not open the link to access the module and 5 did not progress beyond the introductory page (Table 1). Thus, 46 candidates disengaged before any exposure to the online materials. As it was explained in the recruitment, candidates were invited to participate in the research if they write their email address in a paper circulated after a presentation by the researcher. One possible explanation could be that those candidates might have provided their email addresses due to the peer pressure effect and had no intention to take part in the research. However, this supposition is not supported by any evidence as interviews were not carried out to clarify such point for two reasons. Firstly the plan was to design cognitive hazard training module which would be delivered online. The lack of such engagement with the online assessment would be an indication of consent withdrawal. Secondly, the aim of the research was to assess the feasibility of using design in the UK surgical training environment. Investigating an initial research drop out, did not further support or address this aim. Hence only reminder emails were planned in the research protocol agreed within the NHS ethical approval with no further contact or interview invitations to protect candidates' rights to withdraw consent.

In total 47 candidates submitted answers to at least one screen and, of those, 33 finished the whole assessment, therefore 14 dropped out after some exposure to the material. Analysis identified those who did dropout were more prominently junior doctors $(50 \% ; 3 / 6)$. Junior doctors were recruited to support the aim to target the materials at SPR level. The data from both the MCQs and interviews indicates, as intended, that the module was too difficult for junior doctors with candidates rating the module difficulty at eight to nine out of ten, thus junior doctor drop out was understandable.

Staff grades' dropout rate was also relatively high, but numbers were small $(40 \% ; 2 / 5)$ as with consultants' $(33 \% ; 5 / 15)$. Consultant dropout was expected as they are a busy group and were invited to check the suitability of the material, however, staff grade dropout is more difficult to explain.

Fortunately, the main target group, SPRs, had a good completion rate (81\%; 7/9 SPR1 and 10/12 SPR2). This illustrated that once exposed to the materials, SPRs were motivated to complete the training, with a clearly reported shift in candidates' attitude as they progressed through the module.
"Initially I felt that this was just a not a waste of time, like unnecessary time, forty minutes to do a small assessment, but then when I was into it I found it all quite interesting. I found it is also slightly difficult because initially I said this is quite a boring anatomy about the Gall Bladder, but it wasn't, it's very high level so it works for registrars of all levels. ' (SPR12, MCQ interview).

$\underline{\text { Module educational benefit }}$

Candidates had a varying level of experience and different sub-speciality interests such as breast, vascular and gastrointestinal surgery trainees. Some trainees were doing the procedure as part of their surgical 
training, but would not later perform laparoscopic cholecystectomy once appointed to consultant. Despite those variations, the overall feedback was overwhelmingly positive. Candidates enjoyed the concentrated yet comprehensive training provided.

"I thought it was much more informative than say sitting there and reading a book for half an hour, forty minutes. So yeah, I think the time investment is valid.” (SPR 3, MCQ interview)

"It was probably even more specific and detailed than we would teach in a course almost." (Consultant 9, MCQ interview)

SPRs appreciated the focus on risks and anatomical variations which is more relevant to their training level.

"Most people know the steps and how to do it but it's the complications and the Anatomical variations that is what you need to be aware of." (SPR 5, MCQ interview).

'I'm not experienced yet. So, to be able to know exactly what to look for, and then see a video live, an actual recording of how it's done and what can go wrong is very useful. " (SPR 15, MCQ interview)

Even consultants reported a good personal learning value from the online module.

"Even as a surgeon with some experience I would say there was some things there I did not encounter or encountered a long time previously and it was useful to be reminded about them." (Consultant 6, MCQ interview)

"It was good, it was educational, and I learnt stuff." (Consultant 3, MCQ interview)

Candidates enjoyed the real operation video clips with the immediate feedback provided in the module and reported a high degree of validity and simulation to real training environment.

"To see a video that has commentary, essentially, it would simulate if you were doing that operation and your Consultant was telling you at the same time, and I think, yes, that's a good substitute for it." (SPR 6, MCQ interview) 
The benefit of this cognitive training was mainly to highlight rare but dangerous hazards and mentally train candidates to identify hazard clues and avoid errors which would cause injury to patients. This was nicely compared by one candidate to pilot simulation training.

"You have the same comfort the airline pilot has in a simulator... This is a rapid take through a lot of things that could go wrong, problems that you could face, injuries you could face in laparoscopic cholecystectomy. It would take a lifetime to cover all these complications in your practice because they don't happen all the time. This is the same reason airline pilots train in simulators. They probably never have two engines fail on a four-engine aircraft but they have to be trained just in case, so you don't wait for it to happen. " (Consultant 6, MCQ interview)

\section{$\underline{\text { Potential change in practice }}$}

The study aim was to illustrate the potential of the cognitive hazard training module to enhance awareness and knowledge, it would be very difficult for any educational intervention to claim clinical effect, and to do so would require a more complex and longitudinal study design. However, five of the 16 SPRs interviewed explicitly mentioned a change in their operating approach following the online training and two more candidates hinted at such a change. This was a self-reported behavioural change.

"I am now much more conscious of making sure that the critical view of safety is there, I was aware of the concept, I used to apply it but in a very ad hoc way in the past, nowadays I try and dissect everything out thinly, I take a photo of it as well, before I even apply any clips. So it has definitely changed my approach to Lap chole." (SPR 14, MCQ interview)

In this sense, the Cognitive Hazard Training module did not only increase awareness, it possibly induced a behavioural change. Trainees reported implementing the learned principles and such implementation should ultimately lead to better clinical outcomes for their patients. Those trainees are still under supervision and their supervisor would act as a safety net. However, having enhanced awareness should help to accelerate training and reduce the chance of unintended injuries.

\section{Building trainers' trust in their trainees}

The current UK training system has lost the old apprentice style training, as consultants train different trainees on a daily basis. This system reduces the trainers' ability to assign a safe and appropriate training opportunity on the basis of established knowledge about the trainee's ability. As a result, some consultants 
expressed the possible reassurance of knowing that their SPRs had such cognitive hazard training and the module potential to provide a common ground to discuss such topics if needed.

"Yes, I would prefer someone to have done it because it then would give me some common ground to talk about things and to understand what they knew a little bit more." (Consultant 9, MCQ interview)

Candidates appreciated that this module is not a replacement for real operative experience but errors might be safely learned outside theatre to avoid patient harm.

"I would feel happier knowing the SPR done this, I think discovering potential traps, in real time in an actual patient is very useful, but possibly dangerous. Each time you go in you have already built up theoretical knowledge and virtual experience from this teaching package. You have to learn your own lessons but they would have been further improved by learning the lessons of others.” (Consultant 6, MCQ interview)

Knowing such hazard training already took place before operating would potentially enhance trainer trust in their trainees with a potential increase in training opportunity allocation.

\section{Discussion and conclusion}

The overall feedback from the feasibility study was positive and the Cognitive Hazard Training module overcame some of the limitations reported by Wallace et al. review article (14).

The module was suitable for use in the UK surgical training environment as supported by the results of the feasibility study. It was correctly calibrated and targeted at SPRs as shown by the findings from testing with the Foundation Programme doctors and it was unanimously welcomed by all SPRs and consultants. This target group were motivated to complete the training once they have initial exposure to the materials with completion rate of $81 \%$ and positive shift in attitude toward the modules reported in the interviews.

Results supported the value of this online resource in enhancing knowledge and hazard awareness. Interview data also suggested the module's potential to create behavioural modification in the form of cautious dissection and strict adherence to safety steps during the procedure. It also served to support a common language between trainees and trainers which might enhance the trust between trainers and trainees. If this occurred, it would potentially translate into increased dedicated training opportunities which would steepen SPR training curve. Adding such potential enhance training opportunities to the reported practical shift in trainees' approach, the online module would serve a double effect in enhancing training and reducing the time to reach competency. 
The module overcome the high running cost associated with most cognitive surgical training by eliminating the need for expert instructors as it was designed as a dedicated stand-alone online module. The initial steps to creating the module was time consuming and required expertise in the planned operation. However, once the module was up and running the only support needed was general IT support. This feasibility study was acceptable and illustrates that the module implementation costs can be minimal when hosted on an educational website.

\section{$\underline{\text { Study limitation }}$}

This study was conducted to test the feasibility of using cognitive hazard training in the current UK surgical training environment. The study was limited by testing only in one English deanery and with one procedure and with no recruitment selection process.

There was two drop out events. Initial high drop-out was noted before candidates' exposure to the material. such initial drop out could be to peer pressure as candidates provided their email addresses in front of their colleague and had no intention to take part in the research. The lack of such engagement with the online assessment as considered as an indication of consent withdrawal and only reminders were allowed with no further contacts or interview requests planned in the research protocol agreed within the NHS ethical approval to protect candidates' rights to withdraw consent.

After exposure to the first content page a second drop out event took place and were mainly among junior doctors who reported their difficulty in progressing with a module planned for SPRs which is beyond their junior level. It was also noted among consultants which is a busy group and were providing an expert check for material below their level.

Although such drop out did not affect the target group motivation and engagement it would have been useful to investigate such events. Such finding could guide future study design and would help to highlight the need for such dropout investigation to the NHS ethical approval committee and alleviate their objections.

\section{Future dissemination}

This results indicate the feasibility of using a dedicated stand-along online module to deliver cognitive training without the need for instructors. The initial steps to create the module were time consuming and required expertise in the planned operation. However, once the module was up and running the only support needed was general IT support. The research also shows the possible benefit of creating a full cognitive hazard training curriculum in a variety of practical procedures such as endoscopy, minor and major operations. This makes the module implementation cost minimal and the website could be hosted at the ISCP or the Royal Colleges' websites.

Detailed description of the process followed in creating the module and processing the material used is provided in publicly available $\mathrm{PhD}$ thesis (15). The research was part of a $\mathrm{PhD}$ study. The YouTube videos and the image used in the module were obtained under limited permission for the $\mathrm{PhD}$ research use. Hence 
the material cannot be shared without gaining further permission from the copyright holders. However the researcher does not claims any personal copyright and is happy to cooperate with any future research in this field.

\section{References:}

1. Chikwe J, de Souza AC, Pepper JR. No time to train the surgeons. BMJ. 2004;328(7437):418-9.

2. Heath RM, Gate TCS, Halloran CM, Callaghan M, Paraoan MT, Blair SD. The EWTD 'triple whammy': hitting surgical trainees where it hurts. Bulletin of The Royal College of Surgeons of England. 2007;89(1):26-8.

3. Crofts TJ, Griffiths JMT, Sharma S, Wygrala J, Aitken RJ. Surgical training: an objective assessment of recent changes for a single health board. BMJ. 1997;314(7084):891.

4. Cuschieri A. Whither minimal access surgery: tribulations and expectations. American journal of surgery. 1995;169(1):9-19.

5. Stiff GJM, Clarke D, Torkington J, Bowrey DJ, Mansel RE. Training in the Calman era: what consultants say. Bulletin of The Royal College of Surgeons of England. 2002;84(10):345-7.

6. Kneebone R. Simulation in surgical training: educational issues and practical implications. Med Educ. 2003;37(3):267-77.

7. Gawande AA, Zinner MJ, Studdert DM, Brennan TA. Analysis of errors reported by surgeons at three teaching hospitals. Surgery. 2003;133 (6):614-21.

8. Feinmann J, Templeton SK. Arrogant surgeons 'risk another Bristol babies scandal'. The Sunday Times. 20063 September 2006.

9. Hunter DR. Risk Perception and Risk Tolerance in Aircraft Pilots. (DOT/FAA/AM-02/17) ed. Washington DC: Federal Aviation Administration2002.

10. Kahneman D. Thinking, Fast and Slow: Farrar, Straus and Giroux; 2011.

11. Dror IE, Stevenage SV, Ashworth ARS. Helping the cognitive system learn: exaggerating distinctiveness and uniqueness. Applied Cognitive Psychology. 2008;22(4):573-85.

12. Pronin E, Lin DY, Ross L. The Bias Blind Spot: Perceptions of Bias in Self Versus Others. Personality and Social Psychology Bulletin. 2002;28(3):369-81.

13. Dror I. A novel approach to minimize error in the medical domain: Cognitive neuroscientific insights into training*. Medical Teacher. 2011;33(1):34-8.

14. Wallace L, Raison N, Ghumman F, Moran A, Dasgupta P, Ahmed K. Cognitive training: How can it be adapted for surgical education? The Surgeon. 2017;15(4):231-9.

15. ISREB S. Enhancing surgical training by audio-visual simulation with hazard cognitive training and reflection tools: a design-based study in laparoscopic cholecystectomy [e-thesis]. Durham university website: Durham University; 2017.

16. Balija M, Huis M, Nikolić V, Štulhofer M. Laparoscopic Visualization of the Cystic Artery Anatomy. World Journal of Surgery. 1999;23(7):703-7.

17. Braun V, Clarke V. Using thematic analysis in psychology. Qualitative Research in Psychology. 2006;3(2):77101. 